International Journal of Wireless \& Mobile Networks (IJWMN) Vol. 5, No. 3, June 2013

\title{
EFFECTS OF FILTERS ON THE PERFORMANCE OF DVB-T RECEIVER
}

\author{
AKM Arifuzzaman ${ }^{1}$, Rumana Islam ${ }^{1}$, Mohammed Tarique $^{2}$, and \\ Mussab Saleh Hassan ${ }^{2}$ \\ ${ }^{1}$ Department of Electrical and Electronic Engineering \\ American International University-Bangladesh, Banani, Dhaka, Bangladesh \\ E-mail:arifuzzaman, rumana@aiub.edu \\ ${ }^{2}$ Department of Electrical Engineering \\ Ajman University of Science and Technology, Fujairah, UAE \\ E-mail:m.tarique, m.mohammadeajman.ac.ae
}

\begin{abstract}
Digital Video Broadcasting-Terrestrial $(D V B-T)$ is an international standard for digital television services. Orthogonal Frequency Division Multiplexing (OFDM) is the core of this technology. OFDM based system like DVB-T can handle multipath fading and hence it can minimize Inter Symbol Interference (ISI). DVB-T has some limitations too namely large dynamic range of the signals and sensitivity to frequency error. In order to overcome these limitations DVB-T receivers should be optimally designed. In this paper we address the issues related to optimal DVB-T receiver design. There of several signal processing units in a DVB-T receiver. A low-pass filter is one of them. In this paper, we consider some classic filters namely Butterworth, Chebyshev, and elliptic in the DVB-T receiver. The effects of different filters on the performances of DVB-T receiver have been investigated and compared in this paper under AWGN channel condition.
\end{abstract}

\section{KEYWORDS}

DVB, DVB-T, multi-carrier, orthogonal, FFT, IFFT, BER, ISI, AWGN, Butterworth, Elliptic, Chebyschev, $P S D$

\section{INTRODUCTION}

Television (TV) is considered as the most cost-effective source of entertainment, education, and information [1-2]. The International Telecommunication Union (ITU) has estimated $1,416,338,245$ TV sets in the households around the World [3]. Originally TV was introduced as an electromechanical system [2,4]. An all-electronic system was developed in Europe in the twentieth century. During this time period the Federal Communication Commission (FCC) adopted the recommendations made by the National Television System Committee (NTSC) system in USA [3]. Later on a number of color television systems were introduced. The Sequential Couleur A Memoire (SECAM) and Phase Alternating Line (PAL) were introduced in Europe. NTSC color television was introduced in USA. The digital television network merged with the advancements of digital technology. Broadcasters and manufacturer realized the importance of switching from analog to digital television. Digital television became popular because of its improved spectrum efficiency. The television broadcasters could accommodate more channels in a limited spectrum. Several digital TV standards were rapidly developed in the next few years. In Europe broadcasters and electronic companies formed consortium and they introduced Digital Video Broadcasting (DVB). It is a set of internationally recognized standards for digital television services. It is maintained by DVB project [5]. This project is an industryled consortium of more than 200 manufacturers, broadcasters, network operators, software developers, and regulatory bodies. European Broadcasting Union, European Committee for 
International Journal of Wireless \& Mobile Networks (IJWMN) Vol. 5, No. 3, June 2013

Electrotechnical Standardization, and Joint Technical Committee of European Telecommunication Standards Institute publish and update this standard. This standard has been proposed to support digital television services to the consumers by means of rooftop antennas. There are three key DVB standards that define the physical and data link layer of the distribution system. These are (i) DVB-S for satellite network, (ii) DVB-C for cable networks, and (iii) DVB-T for terrestrial networks [6-8]. There are also some related standards. Some of these are DVB-H and DVB-IPTV. The DVB-H has been standardized to support mobile television services in handheld devices. On the other hand, DVB-IPTV has been standardized to support television service by using Internet Protocol (IP) [9]. Among all these standards DVB-T system has become the most popular one and is the topic of this investigation. DVB-T system has been formally defined and introduced in [10]. The system is designed to operate within the existing Very High Frequency Band (50-230 MHz) and Ultra High Frequency Band (470-870 $\mathrm{MHz}$ ). Two modes of operation have been defined for DVB-T standard namely " $2 \mathrm{~K}$ mode" and " $8 \mathrm{~K}$ mode". The " $2 \mathrm{~K}$ mode" has been defined for DVB-T transmission and the " $8 \mathrm{~K}$ mode" has been defined for DVB-H transmission [11]. For single transmission and limited distance operation the " $2 \mathrm{~K}$ mode" is considered suitable. On the other hand, the " $8 \mathrm{~K}$ mode" is preferable for long distance operation. The DVB-T standard allows different levels of Quadrature Amplitude Modulation (QAM) and it uses different inner code rates. The system also allows two levels of hierarchical channel coding and modulation. The basic functional block diagram of DVB standard is shown in Figure 1. The processes applied to the input data stream are (i) transport multiplex adaptation, (ii) outer coding, (iii) outer interleaving, (iv) inner coding, (v) inner interleaving, (vi) mapping and modulation, and (vii) OFDM transmission.

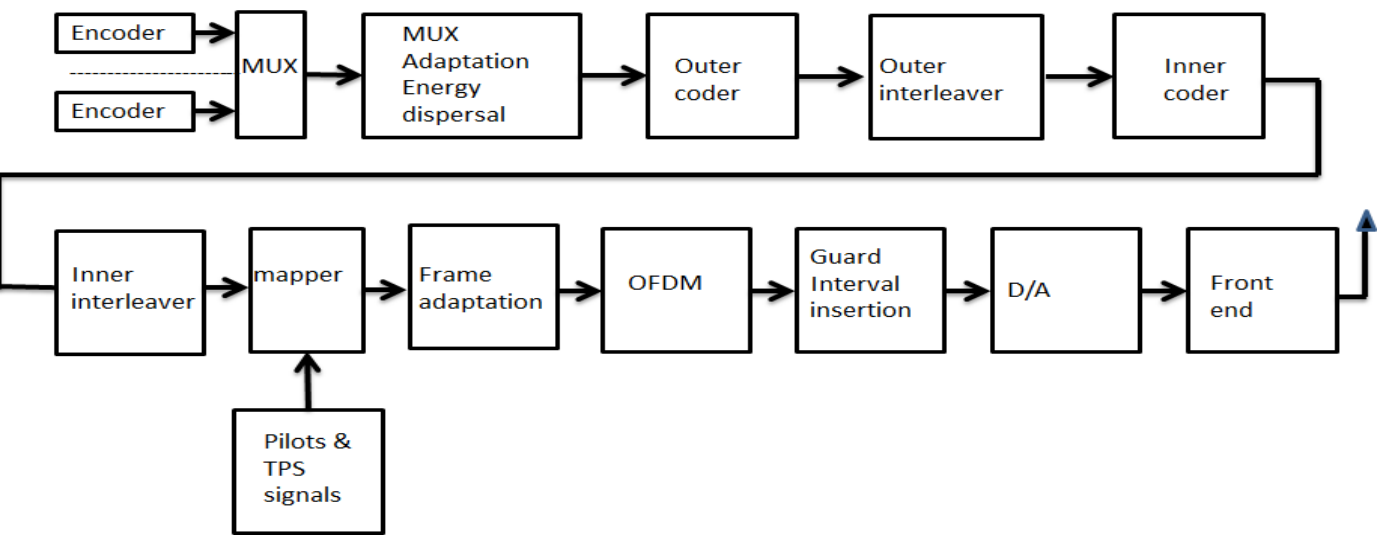

Figure 1. Block ddiagram of DVB system.

Most of the signal processing steps shown in Figure 1 is carried out in a digital signal processor. The performance of DVB system highly depends on the modulation. Since this investigation addresses the physical layer issues the basic components we investigate here are the OFDM, Digital-to-Analog Converter (D/A) and the front end. Brief descriptions of these components are provided here for the completeness of this paper.

\section{OFDM MODULATION}

The Orthogonal Frequency Division Multiplexing (OFDM) is a multi-carrier modulation (MCM) scheme [12-13]. The main principle of MCM is to divide the high rate input bit stream into several low rate parallel bit streams and then these bit streams are used to modulate several sub-carriers. The sub-carriers are separated by a guard band to ensure that they do not overlap with each other during the course of transmission. In the receiver side band-pass filters are used to separate the spectrum of individual sub-carriers. OFDM is a special form of spectrally efficient MCM technique, which employs densely spaced orthogonal sub-carriers and overlapping spectrums. Hence, it is considered as a spectrum efficient modulation scheme. 
International Journal of Wireless \& Mobile Networks (IJWMN) Vol. 5, No. 3, June 2013

Unlike other systems OFDM does not require band-pass filters because of the orthogonality nature of the subcarriers. Hence the available bandwidth is used very efficiently without causing the Inter Carrier Interference (ICI). The required bandwidth can be further reduced by removing the guard band and allowing the subcarriers to overlap. Despite the overlapping spectrum it is still possible to recover the sub-carriers provided that the orthogonality condition among the subcarriers is maintained. OFDM provides a composite high data rate with long symbol duration. Depending on the channel coherence bandwidth OFDM reduces or completely eliminates the effect of Inter Symbol Interference (ISI). The Discrete Fourier Transform (DFT) and Inverse Discrete Fourier Transform (IDFT) can be used to implement the orthogonal signals. OFDM has numerous advantages over other digital modulation schemes. The main advantage of OFDM is that it can minimize the Inter Symbol Interference (ISI), which is originated from the multipath signal propagation [14] as mentioned earlier. Hence, it can support a very high data rate. There are some other advantages too. OFDM can cope with the multipath fading with less computational complexity compared to other modulation schemes. It is more resistant to frequency selective flat fading. It uses computationally efficient Fast Fourier Transform (FFT) and Inverse Fast Fourier Transform (IFFT) algorithms. OFDM is also considered suitable for coherent demodulation. Additionally, OFDM can ensure the required Quality of Service (QoS) to the end users depending on the bandwidth. It is also considered suitable for diversity techniques (i.e., time diversity and frequency diversity). The orthogonality of subcarriers of an OFDM system can be jeopardized when the same is sent through the multipath channel, which also introduces Inter Carrier Interference (ICI). Cyclic Prefix (CP) is added with the OFDM symbol to combat both ISI and ICI. The CP contains a copy of the last part of the OFDM symbol appended to the front of transmitted OFDM symbol. The length of the $\mathrm{CP}$ must be longer than the maximum delay spread of the multipath environment to reduce the ISI. OFDM has some disadvantages too. Some of these are: (i) it requires a strong synchronization between the transmitter and the receiver, (ii) it is very much sensitive to phase noise and frequency offset, (iii) it is not power efficient because of the always active Fast Fourier Transform (FFT) algorithm and Forward Error Correction (FEC), (iv) it cannot take the advantages of the diversity gain if few sub-carriers are allotted to each user, and (v) it requires a high peak to average ration RF power amplifiers to avoid amplitude noise. There have been numerous works carried out to overcome these disadvantages. We have included some of them here that are related to this work.

\section{RELATED WORKS}

The bandwidth efficiency of OFDM systems is originated from the overlapping orthogonal subcarriers. Traditionally it is assumed that the waveforms of OFDM transmission have band limited and the spectrums overlap only with its adjacent sub-carrier. But, in real life scenario the OFDM transmission is not band limited. Hence, the spectrums not only interfere with the adjacent carrier, but they also interfere with the further-away sub-carriers [15]. To limit this type of interference the spectrum of OFDM transmission must be kept in a sufficiently low stopband. Many research works and innovations have been done to minimize the interference. One of the early solutions has been presented in [16]. The authors suggested some analysis which led to use truncated Prolate Spheroidal Wave Function (PSWF) [17]. Non-linear programming technique has been used to design FIR filters for transmitter and receiver in [18]. Pulse shaping and filtering have been suggested to limit the bandwidth of OFDM symbol. Raised Cosine Function (RCF) has been used in [19-22] to reduce the inter symbol interference (ISI) in OFDM system. The authors have shown in these works that BER can be reduced by using RCF filters. It is shown in [23] that reconstruction filters, anti-aliasing filters and other filters cause smearing in an OFDM system. The author presented some numerical works in the same work and they suggested that Chebyshev II filter approximations should be the best choice. This type of filter causes the least smearing. A series of investigations also shows that filtering is important to reduce Peak-to-Average Ration (PAR) of OFDM system. The authors suggested in [24] that the 
International Journal of Wireless \& Mobile Networks (IJWMN) Vol. 5, No. 3, June 2013

clipping is the simplest method to reduce PAR reduction. But, it causes both in-band distortion and out of band distortion. Hence, a filter should be used after the clipping to reduce the distortions. A more efficient method for clipping and filtering has been proposed in [25,26]. In these works the authors demonstrated that the clipping and filtering algorithm are better than clipping along to reduce PAPR in OFDM system. An easy to implement method of clipping and filtering has been discussed in [27]. The effects of performing channel estimation of an OFDM system by using non-ideal interpolating and decimating filters have been investigated under both AWGN and Rayleigh fading channel in [28]. Two schemes for ICI reduction have been proposed in [29]. In self-cancellation scheme redundant data is transmitted onto adjacent subcarriers to reduce ICI. In the second scheme an Extended Kalman Filter (EKF) corrects the frequency offset at the receiver. In a related work [30] an unscented Kalman filter based solution has also been provided. In contrast to other related works the authors considered high mobility OFDM system in this work. The effects of imperfect anti-aliasing filtering have been investigated in this paper [31]. The authors have used a linear phase FIR filter of order $\mathrm{N}=50$ in this investigation. In [32] it has been claimed that pulse shaping filter is not good enough for ICI cancellation in OFDM system. A hybrid scheme consisting of pulse shaping and Maximum Likelihood Estimation (MLE) should be combined to cancel ICI. In [33] a non-linear adaptive filter has been proposed. The authors claimed that non-linear filter scheme is superior to the conventional linear filter.

In all the above mentioned works the investigators focused on a particular filter to improve the performance of OFDM system. Only a few works provided a comparative performance analysis of different types of filter. In these work we considered some classic filters namely Butterworth filter, Elliptic filters, and Chebyshev filters. We varied some filter parameters like cut-off frequency and filter order to investigate the performance of DVB-T receiver. It is worthwhile to mention another difference here. In all the above mentioned works basic OFDM system has been used. In this paper we consider DVB-T system, which uses OFDM as a modulation scheme.

\section{DVB-T FRAME STRUCTURE}

The DVB-T transmitted signals are organized into frame structure. Each frame consists of 68 OFDM symbols [9]. Since OFDM is the core of DVB-T system, we will use DVB-T symbol and OFDM symbol interchangeably in the remaining portion of this paper. Each OFDM symbol consists of a set of $\mathrm{K}=1705$ carriers in the " $2 \mathrm{~K}$ mode" and $\mathrm{K}=6817$ carriers for " $8 \mathrm{~K}$ mode". Let us denote the frame duration by $\mathrm{T}_{\mathrm{F}}$ and the symbol duration by $\mathrm{T}_{\mathrm{S}}$. The symbol duration has two components namely a useful part and a guard interval. Let us denote the useful component by $\mathrm{T}_{\mathrm{U}}$ and the guard component by $\mathrm{T}_{\mathrm{G}}$. The guard interval carries cyclic prefix $(\mathrm{CP})$ inserted before the useful component (as mentioned earlier). Four guard intervals have been chosen in DVB-T standard namely $1 / 4,1 / 8,1 / 16$ and $1 / 32$. The symbols of OFDM are numbered from 0 to 67 . In DVB-T standard all symbols contain data and reference information. The OFDM symbol can be considered as a combination of many separately modulated carriers. Hence each symbol can be considered to be divided into cells. The transmitted data of an OFDM frame contains: (i) scattered pilot cells, (ii) continual pilot carriers, and (iii) Transmission Parameter Signaling (TPS) carriers. The pilots are used for frame synchronization, frequency synchronization, time synchronization, channel estimation, and transmission mode identification. The TPS carriers are used for the purpose of signaling parameters (i.e., channel coding and modulation) related to the transmission scheme, The carriers are indexed from $K_{\min }$ to $K_{\max }$, where $K_{\min }=0$ and $K_{\max }=1704$ for " $2 \mathrm{~K}$ mode" and $\mathrm{K}_{\max }=6816$ in " $8 \mathrm{~K}$ mode". The spacing between $\mathrm{K}_{\min }$ and $\mathrm{K}_{\max }$ is defined by $(\mathrm{K}-1) / \mathrm{T}_{\mathrm{U}}$, where the spacing between adjacent carriers is $1 / \mathrm{T}_{\mathrm{U}}$ and $\mathrm{K}$ is the number of the carriers. The numerical value for the OFDM parameters for the " $2 \mathrm{~K}$ mode" and " $8 \mathrm{~K}$ mode" are listed in Table 1. 
Table 1. OFDM Parameters for " $2 \mathrm{~K}$ " and " $8 \mathrm{~K}$ " mode

\begin{tabular}{|l|l|l|}
\hline Parameter & $8 \mathrm{~K}$ mode & $2 \mathrm{~K}$ mode \\
\hline Number of carriers $\mathrm{K}$ & 6817 & 1705 \\
\hline $\begin{array}{l}\text { Value of carrier number } \\
\mathrm{K}_{\min }\end{array}$ & 0 & 0 \\
\hline $\begin{array}{l}\text { Value of carrier number } \\
\mathrm{K}_{\max }\end{array}$ & 6816 & 1704 \\
\hline Duration $\mathrm{T}_{\mathrm{U}}$ & $896 \mu \mathrm{s}$ & \\
\hline Carrier spacing $1 / \mathrm{T}_{\mathrm{U}}$ & $1116 \mathrm{~Hz}$ & $224 \mu \mathrm{s}$ \\
\hline $\begin{array}{l}\text { Spacing between } \mathrm{K}_{\min } \text { and } \\
\mathrm{K}_{\max }(\mathrm{K}-1) / \mathrm{T}_{\mathrm{U}}\end{array}$ & $761 \mathrm{MHz}$ & $4464 \mathrm{~Hz}$ \\
\hline
\end{tabular}

The transmitted OFDM symbol can be mathematically expressed by

$$
s(t)=\operatorname{Re}\left\{e^{2 \pi f_{c} t} \sum_{m=0}^{\infty} \sum_{l=0}^{67} \sum_{k=K_{\min }}^{K_{\max }} c_{m, l, k} \times \psi_{m, l, k}(t)\right\}
$$

where

$$
\begin{aligned}
& \psi_{m, l, k}(t)= \begin{cases}e^{j 2 \pi \frac{k^{\prime}}{T_{U}}\left(t-T_{G}-l \times T_{S}-68 \times m \times T_{S}\right.} & (l+68 \times m) \times T_{S} \leq t \leq(l+68 \times m+1) \times \\
\psi_{m, l, k}(t)=0 \text { else }\end{cases}
\end{aligned}
$$

where $k=$ the carrier number, $l=$ the OFDM symbol number, $m=$ the frame number, $K=$ the number of transmitted carrier, $f_{c}=$ the central frequency, $k \square=$ the carrier index related to the canter frequency, and $\mathrm{c}_{\mathrm{m}, \mathrm{l}, \mathrm{k}}=$ complex symbol for carrier $k$ of the data symbol number $l$ in frame $m$. The $c_{\mathrm{m}, \mathrm{l}, \mathrm{k}}$ values are normalized according to the constellation points of the modulation

\begin{tabular}{|c|c|c|c|c|c|c|c|c|}
\hline Mode & \multicolumn{4}{|c|}{$8 \mathrm{~K}$ mode } & \multicolumn{4}{|c|}{$2 \mathrm{~K}$ mode } \\
\hline Guard interval $\mathrm{T}_{\mathrm{G}} / \mathrm{T}_{\mathrm{U}}$ & $1 / 4$ & $1 / 8$ & $1 / 16$ & $1 / 32$ & $1 / 4$ & $1 / 8$ & $1 / 16$ & $1 / 32$ \\
\hline Duration of symbol part $T_{U}$ & & & & $\begin{array}{r}8192 \times \mathrm{T} \\
=896 \mu \mathrm{s}\end{array}$ & & & & $\begin{array}{r}2048 \times \mathrm{T} \\
=224 \mu \mathrm{s}\end{array}$ \\
\hline Duration of Guard Interval $\mathrm{T}_{\mathrm{G}}$ & $\begin{array}{l}2048 \times \mathrm{T} \\
=224 \mu \mathrm{s}\end{array}$ & $\begin{array}{r}1024 \mathrm{xT} \\
=112 \mu \mathrm{s}\end{array}$ & $\begin{array}{r}512 \times \mathrm{T} \\
=56 \mu \mathrm{s} \\
\end{array}$ & $\begin{array}{l}256 \times \mathrm{T} \\
=28 \mu \mathrm{s} \\
\end{array}$ & $\begin{array}{r}512 \times \mathrm{T} \\
=56 \mu \mathrm{s} \\
\end{array}$ & $\begin{array}{r}256 \mathrm{xT} \\
=28 \mu \mathrm{s}\end{array}$ & $\begin{array}{r}128 \times \mathrm{T} \\
=14 \mu \mathrm{s}\end{array}$ & $\begin{array}{l}64 \times \mathrm{T} \\
=7 \mu \mathrm{s}\end{array}$ \\
\hline Symbol Duration $\mathrm{T}_{\mathrm{S}}$ & $\begin{array}{r}10240 \\
\mathrm{xT} \\
1120 \mu \mathrm{s} \\
\end{array}$ & $\begin{array}{l}9216 \times \mathrm{T} \\
1008 \mu \mathrm{s}\end{array}$ & $\begin{array}{r}8704 \times \mathrm{T} \\
952 \mu \mathrm{s}\end{array}$ & $\begin{array}{r}8448 \times \mathrm{T} \\
924 \mu \mathrm{s}\end{array}$ & $\begin{array}{r}2560 \times \mathrm{T} \\
280 \mu \mathrm{s}\end{array}$ & $\begin{array}{r}2304 \times \mathrm{T} \\
252 \mu \mathrm{s}\end{array}$ & $\begin{array}{r}2176 \times \mathrm{T} \\
238 \mu \mathrm{s}\end{array}$ & $\begin{array}{r}2112 \times \mathrm{T} \\
231 \mu \mathrm{s}\end{array}$ \\
\hline
\end{tabular}
alphabet used. The other parameters of DBV system are listed in Table 2.

Table 2. Additional Parameters

\section{DVB TRANSMITTER AND RECEIVER MODEL}

The transmitter and the receiver models proposed in [34] have been used in this investigation. The basic signal processing steps done in the transmitter and the receiver of DVB-T system are shown in Figure 2. Since the number of carriers in DVB-T system is 1705, we considered 1705 4-QAM symbols as the input data to the transmitter. We used a bandwidth of $8.0 \mathrm{MHz}$. The carrier frequency we chose is $90 \mathrm{MHz}$. We divided the $8.0 \mathrm{MHz}$ into 1705 sub-carriers. Since the OFDM symbol duration is specified considering a 2048-point Inverse Fast Fourier Transform (IFFT), we chose 4096-point IFFT. Hence, 2391 zeros were padded with the 
information symbol. The output carriers of IFFT were then converted into continuous time signal in the next signal processing step as shown in Figure 2. This step of the signal processing is accomplished by using a pulse shaping filter and a low pass filter [35] denoted by Digital to Analog (D/A) filter in the block diagram shown in Figure 2(a). The pulse shaping filter converts the discrete time carrier output signals into continuous signal. In the next step a D/A filter with a sharp bandwidth is used. We choose a Butterworth filter for this purpose. Finally, the carrier modulation is performed at the last stage.

Designing an OFDM based DVB receiver has been an open research issue since its conception. Like this work most of the researches conducted and innovations have been done in the DVB receiver design. The DVB receiver model used in this investigation is shown in Figure 2(b).

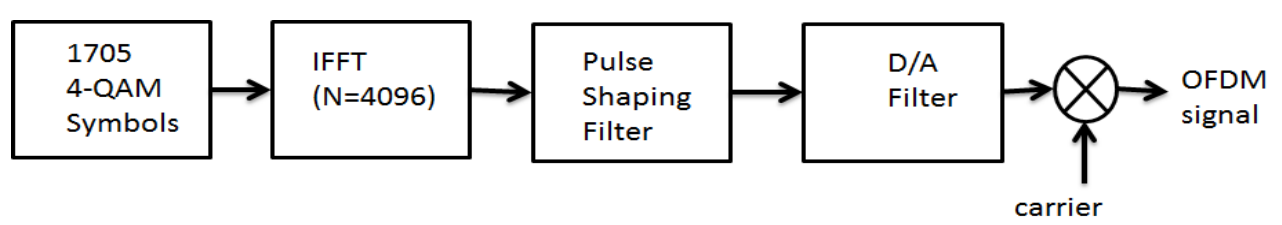

(a) Transmitter model

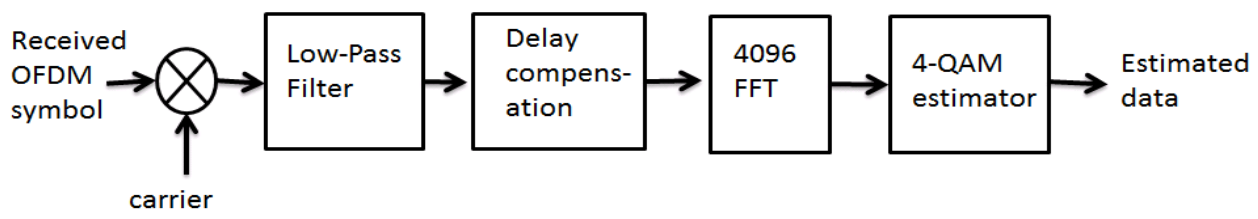

(b) Receiver model

Figure 2. OFDM transmitter and receiver model

The DVB receiver operation performs the inverse operation of the transmitter. First, the coherent demodulation is done. The demodulated OFDM signal is then passed through the lowpass filter to recover the continuous version of the OFDM signal. The delays produced by the reconstruction and demodulation filters are compensated. The resultant signal is then sampled to convert continuous OFDM symbol into its discrete version. The resulted signal is then passed through a 4096-FFT to do the inverse function of its counterpart (i.e., IFFT) done in the transmitter. Finally, the resultant data is estimated by using a 4-QAM estimator. The power spectral density (PSD) of DVB symbol is shown in Figure 3. A numerous investigations show that a significant level of OFDM spectrum falls outside the nominal bandwidth as shown in Figure 3. Hence an appropriate filter should be carefully selected in the transmitter and the receiver to minimize this out of band spectrum. In this investigation three classic filters namely Butterworth filter, Chebyshev filter, and elliptic filters have been considered and the DVB receiver performances have been investigated. 


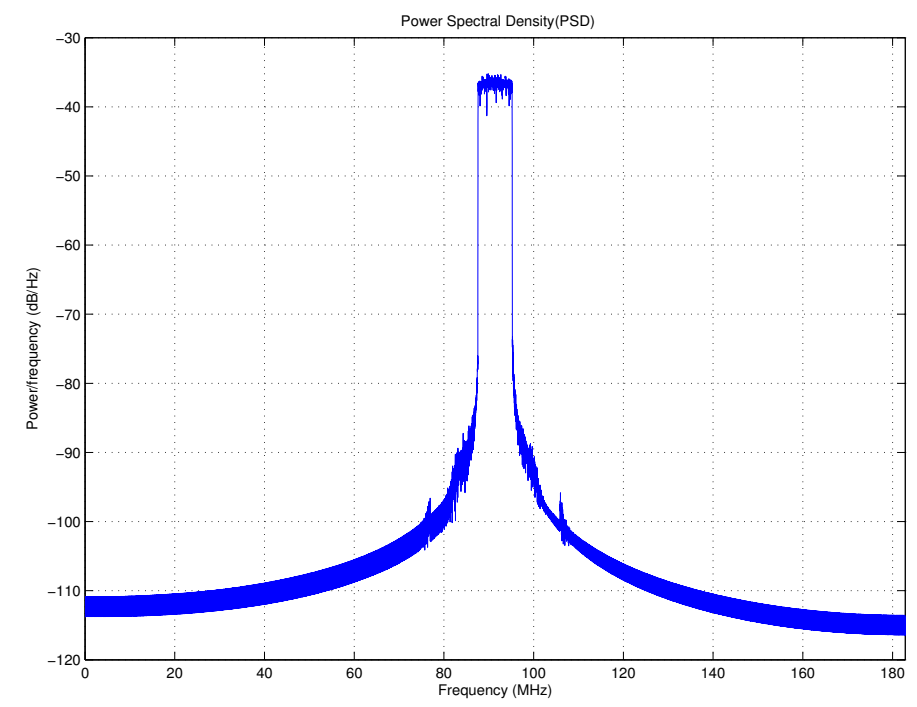

Figure 3. The Power Spectral Density (PSD) of transmitted symbols of DVB.

\section{Classic Filters}

The Butterworth filter has drawn considerable attentions in communication system design. This type of filter exhibits a nearly flat pass band with no ripple in it. The roll off of Butterworth filter is very smooth and monotonic. For a low pass Butterworth filter the roll off rate is $20 \mathrm{~dB} / \mathrm{decade}$ for each added pole in the transfer function. In order to generate the spectrum of different filters we used MATLAB built-in function [36-39]. In MATLAB all digital frequencies are given in units of $\pi$. Hence, the ranges of the cut-off frequencies are defined by $0 \leq w_{c} \leq \pi$. In the rest of this paper we denote the cut-off frequency as $w_{c}=1 / 2,1 / 3,1 / 4$, and $1 / 5$, where $w_{c}=1 / 2$ is the highest cut-off frequency and $w_{c}=1 / 5$ is the lowest cut-off frequency. We selected this frequency arbitrarily for this investigation. The spectrums of Butterworth filters of different orders are shown in Figure 4. In general a higher order filter has higher attenuation rate and vice versa as depicted in the figure. Another important characteristic of Butterworth filter is the cut-off frequency. The spectrums of Butterworth filter with different cut-off frequencies and the same order $\mathrm{N}=3$ are shown in Figure 5. The filters with higher cut-off frequencies have higher attenuation rates as shown in the same figure. The second category filter that is considered in this investigation is the Chebyshev filter. The Chebyshev filter response has a faster roll-off. But, it has ripple in the frequency response. There is a trade-off between ripple and roll-off characteristic of this type of filter. As the ripple increases, the roll-off becomes higher. Like Butterworth filter the spectrums of Chebyshev filter also vary with respect to the order and cut-off frequency. The Chebyshev filters can be classified as Type-I and Type-II. Type-I Chebyshev filter has ripple in the pass band. On the other hand Type-II Chebyshev filter has ripple in the stop band. Since, Type-II filter is rarely used, we consider only Type-I filter in this investigation. Figure 6 shows the spectrums of Chebyshev filter of different order with a pass band ripple of $20 \mathrm{~dB}$ and cut-off frequencies of $180 \mathrm{MHz}$. Figure 7 shows the spectrums of Chebyshev filters with different cut-off frequencies. Compared to Butterworth filter the Chebyshev filter can achieve a sharper transition between the pass band and the stop band with a lower order filter. The sharp transition between the pass band and the stop band of a Chebyshev filter produces smaller absolute errors and faster execution speeds than a Butterworth filter. 
International Journal of Wireless \& Mobile Networks (IJWMN) Vol. 5, No. 3, June 2013

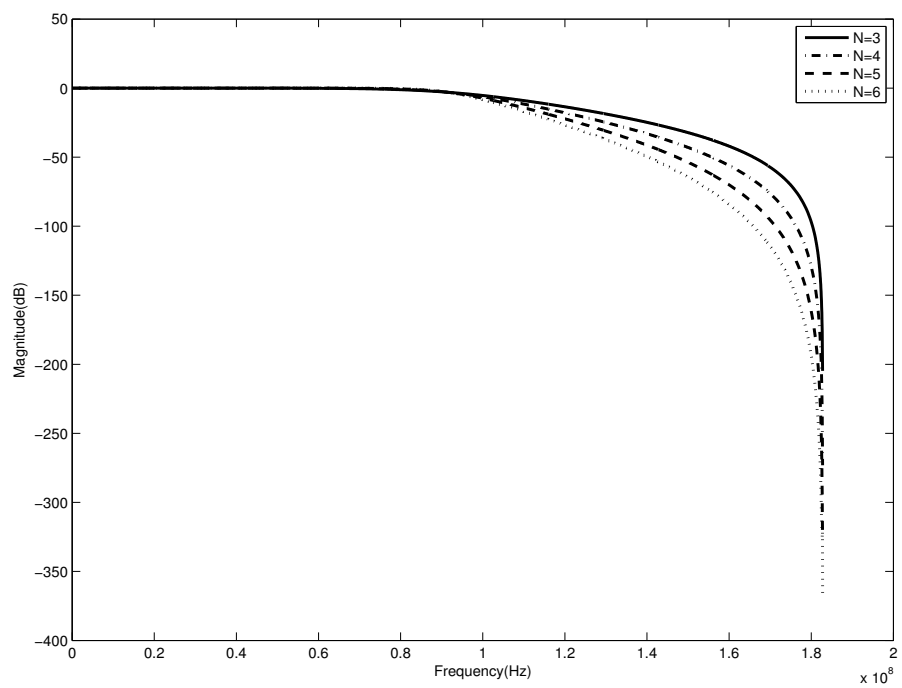

Figure 4. Spectrums Butterworth filters of different orders

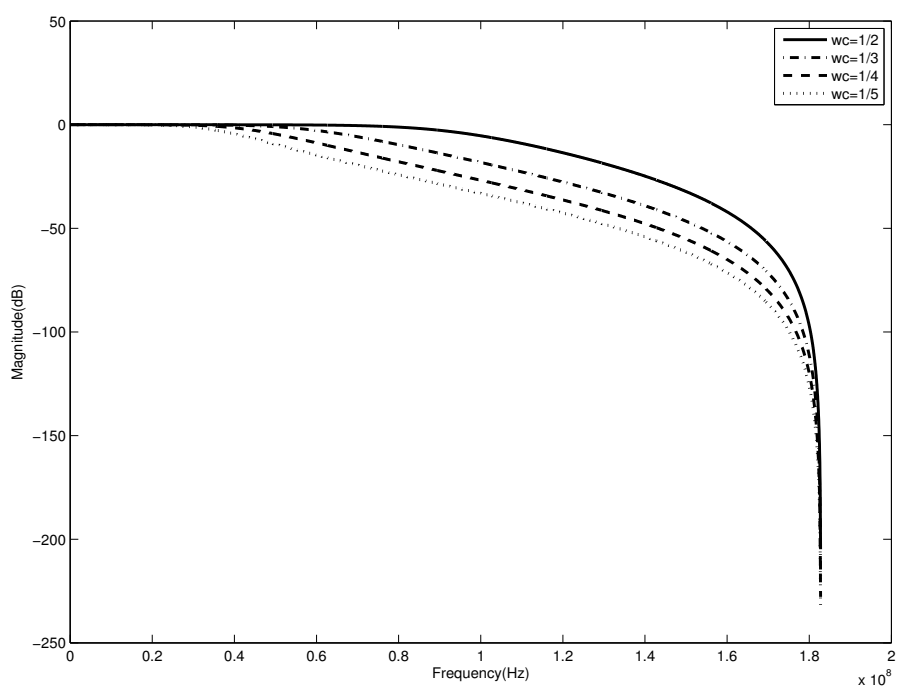

Figure 5. Spectrums of Butterworth filters of different cut-off frequencies 


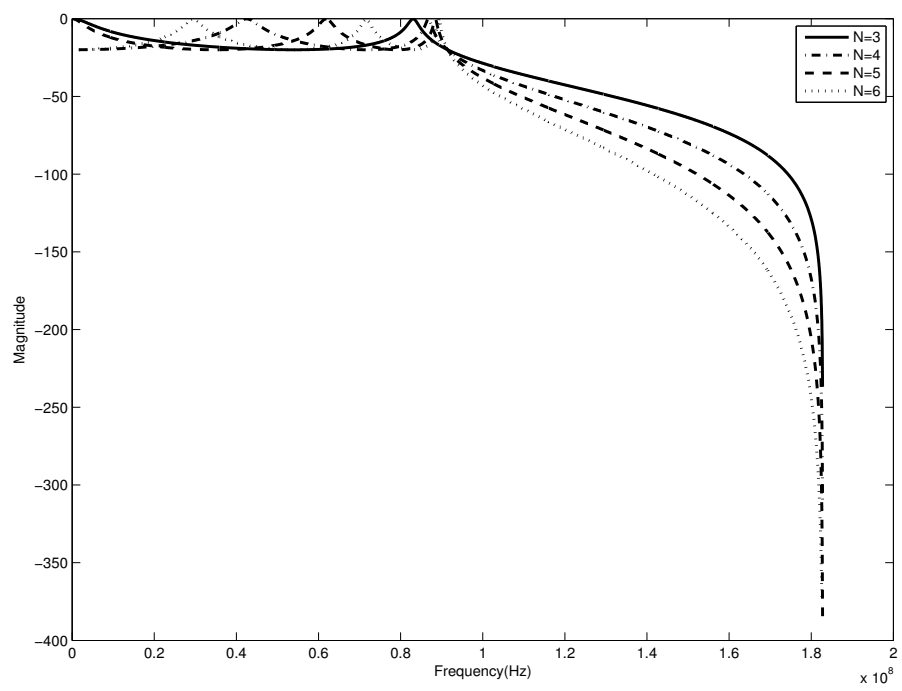

Figure 6. Spectrums of Chebyshev filters of different order

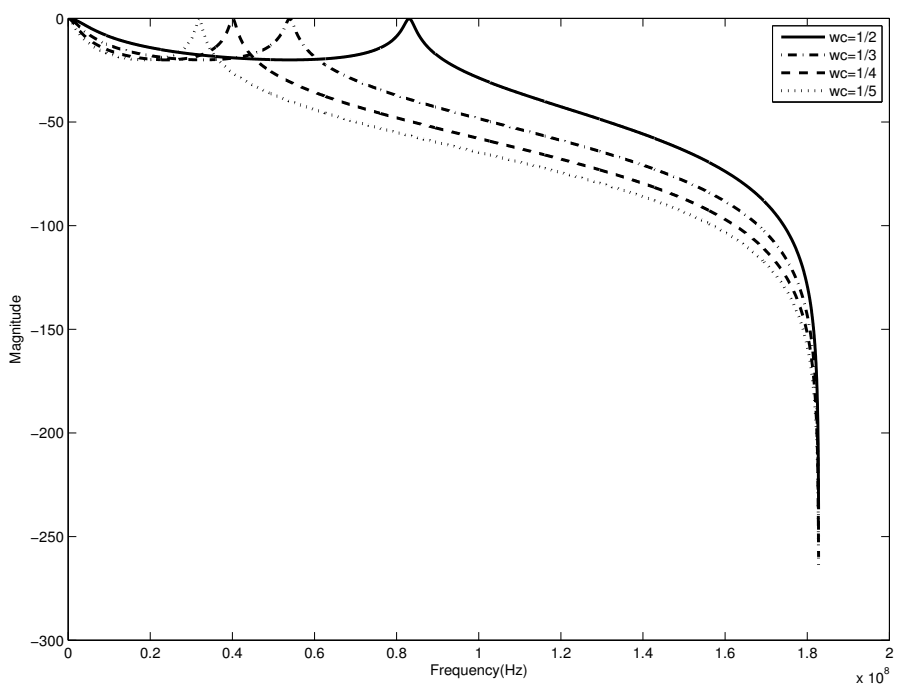

Figure 7. Spectrums of Chebyshev filters of different cut-off frequencies

The last category filter that has been considered in this investigation is the elliptic filter. The cut-off slope of an elliptic filter is steeper than that of a Butterworth and Chebyshev. But, this type of filter has ripple in the stop band. The phase response is very nonlinear. The spectrums of elliptic filters of different orders and cut-off frequencies are shown in Figure 8 and Figure 9 respectively. Compared with the same order Butterworth and Chebyshev filters, the elliptic filters provide the sharpest transition between the pass band and the stop band. 


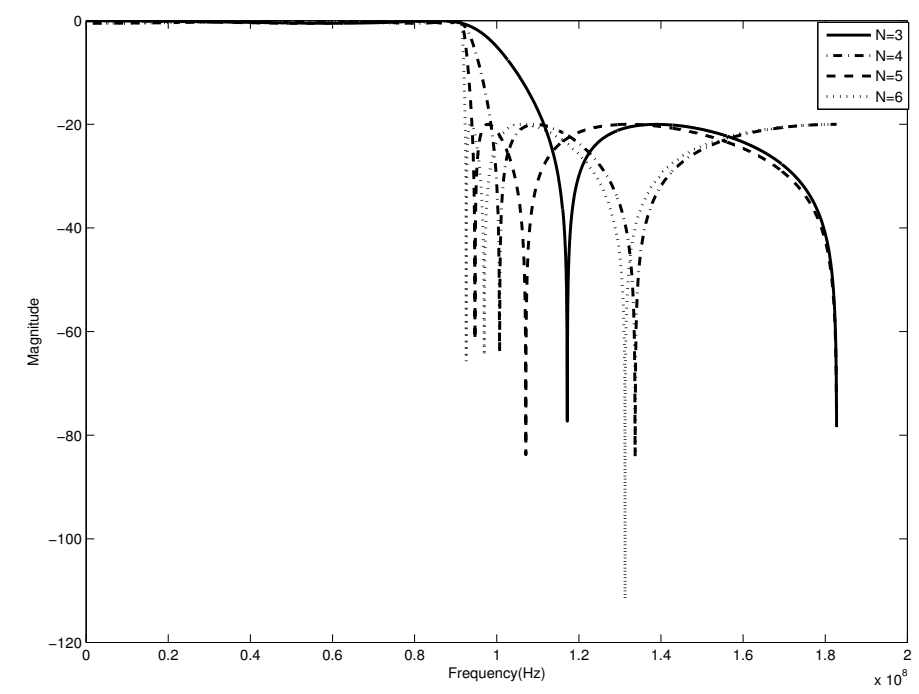

Figure 8. Spectrums of Elliptic filters of different order

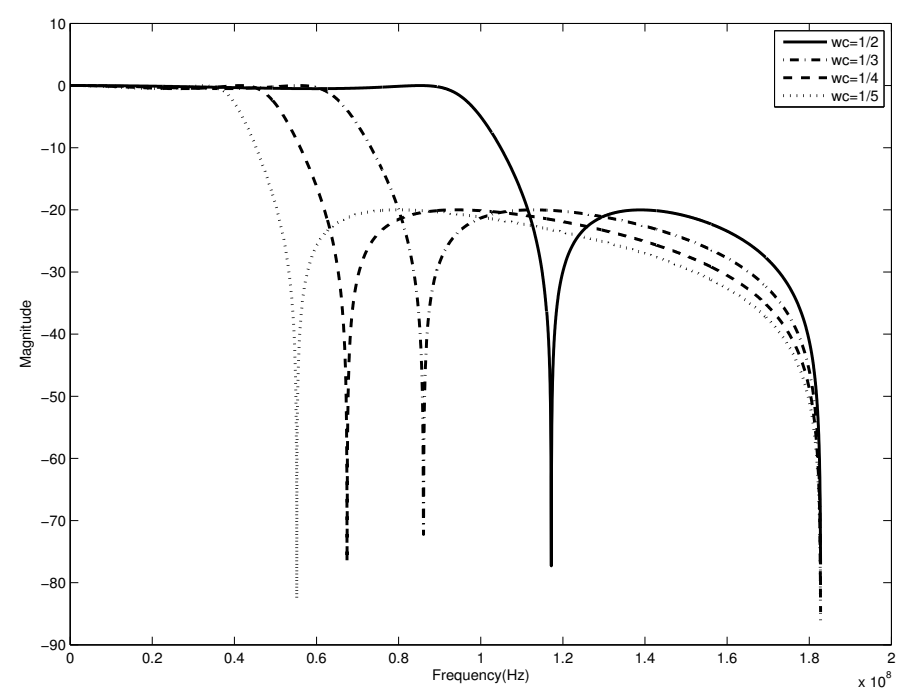

Figure 9. Spectrums of Elliptic filters of different cut-off frequencies

\section{DVB RECEIVER PERFORMANCES USING CLASSIC FILTERS}

Since the effects of digital filters are the main focus in this investigation, the cyclic prefix was set to 0 in all simulations. The other parameters were set to the values as mentioned in Table 1 and Table 2. The channel model was chosen Additive White Gaussian Noise (AWGN). 


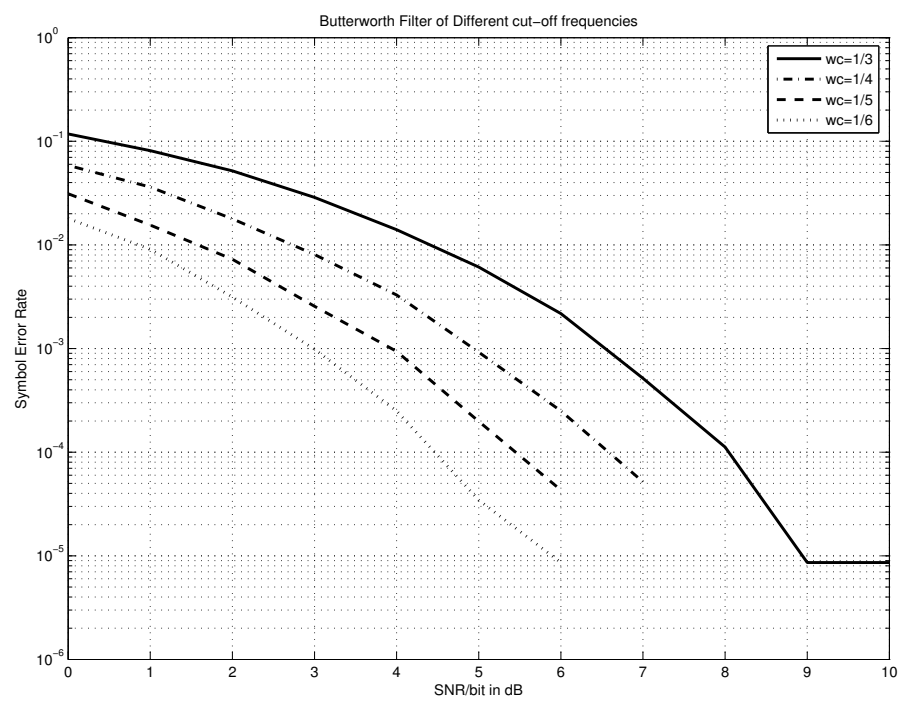

Figure 10. The SER error performances of OFDM system using Butterworth filter (cut-off frequency varied)

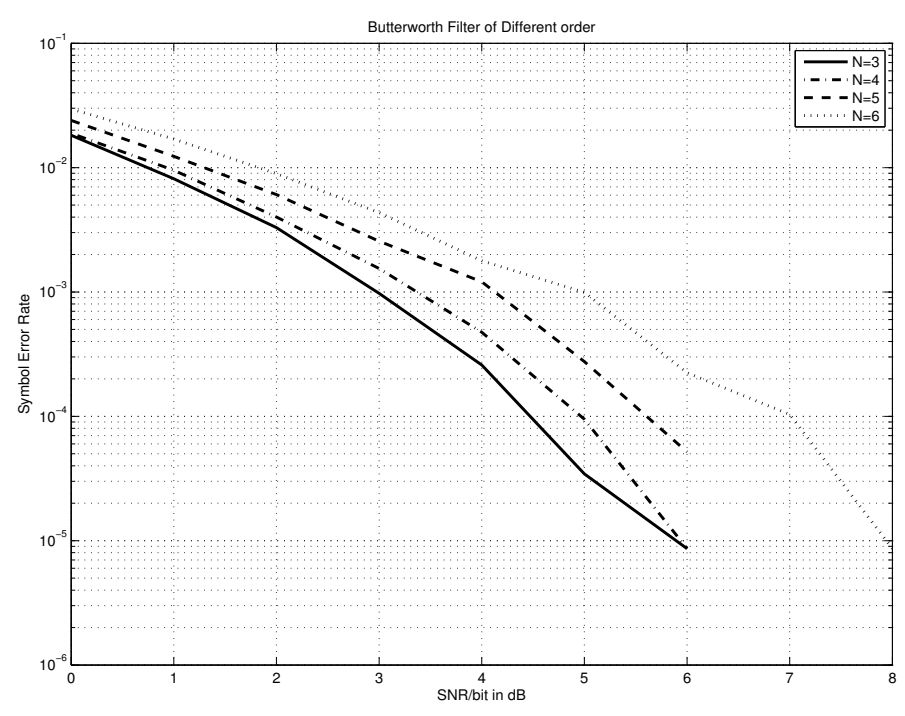

Figure 11. The SER performances of OFDM system using Butterworth filter (order varied)

In the first investigation we consider Butterworth filter in the receiver. The filter order was 3 , but the cut-off frequency was varied. The simulation results are compared in Figure 10. The simulation results show that the lower cut-off frequency shows better performance (i.e. less symbol error rate) than the higher cut-off frequencies. For example, the results show that for a given SNR of $4 \mathrm{~dB}$, the symbol error rate (SER) are 0.091, 0.005, 0.001, and 0.0009 for cut-off frequency of $1 / 3,1 / 4,1 / 5$ and $1 / 6$ respectively. To investigate the performance of OFDM system under varying filter orders we chose a Butterworth filters with cut-off frequency of $\mathrm{w}_{\mathrm{c}}=1 / 5$ and we varied the filter order. The simulation results are shown in Figure 11. The simulation results presented therein show that the lower order Butterworth filters are preferable 
International Journal of Wireless \& Mobile Networks (IJWMN) Vol. 5, No. 3, June 2013

in DVB-T receiver. For a given SNR filter with lower order provides less SER. But, the DVB receiver performance is more dependent on the cut-off frequency other than filter order as illustrated in Figure 10 and Figure 11.

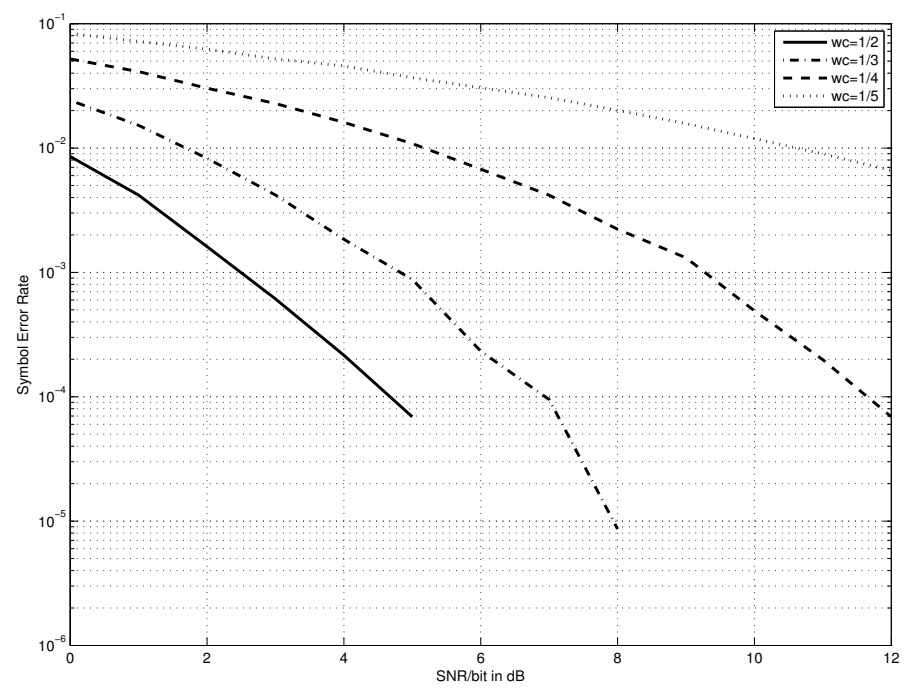

Figure 12. The SER performances of OFDM system using Chebyshev filter (cut-off frequency varied)

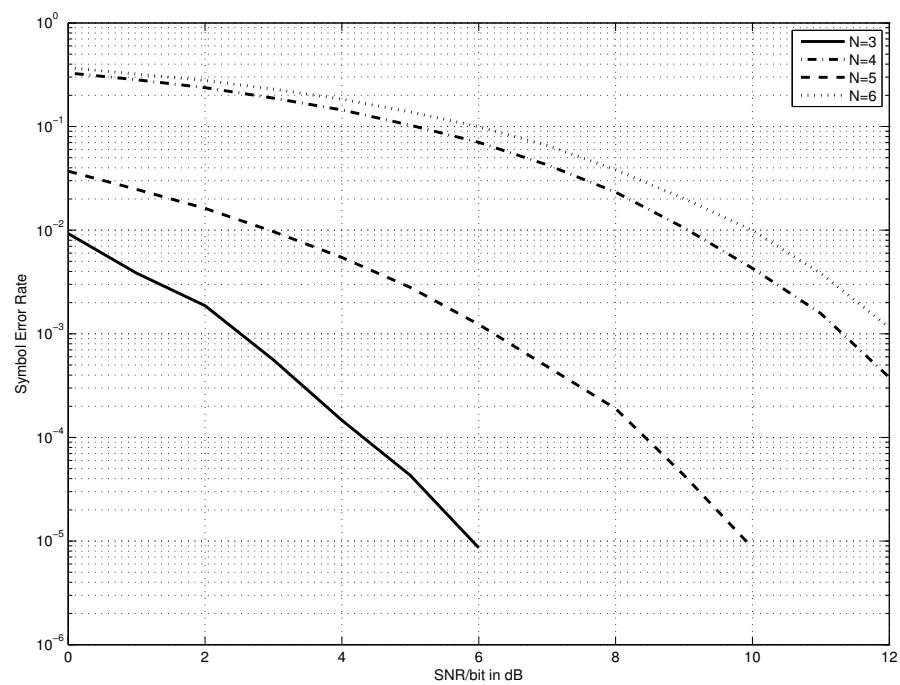

Figure 13. The BER performances of OFDM system using Chebyshev Filter (order was varied)

In the second investigation the receiver filter was then changed to Chebyshev filter by keeping all other parameters same. The filter order was set to 3 and the cut-off frequency was varied. The pass band ripple was set to $10 \mathrm{~dB}$. The simulation results are shown in Figure 12. In contrast to Butterworth filter the simulation results show that Chebyshev filters with higher cut- 
off frequencies are desirable for DVB-T receiver. For a given SNR Chebyshev filter caused less SER compared to Butterworth filter. For an example, the results show that when SNR was set to $4 \mathrm{~dB}$, the symbol error rate (SER) are 0.04, 0.02, 0.002, and 0.0002 for cut-off frequencies $1 / 3$, $1 / 4,1 / 5$ and 1/6 respectively. Compared to those of Butterworth filter it is evident that SER is less in Chebyshev filter. For an example, In the next investigation the filter cut-off frequency was kept constant at $1 / 2$, but the filter order was varied. The simulation results are shown in Figure 13. The results show that a low filter order performs better than a higher order filter. The performance variation is similar to that of Butterworth filter.

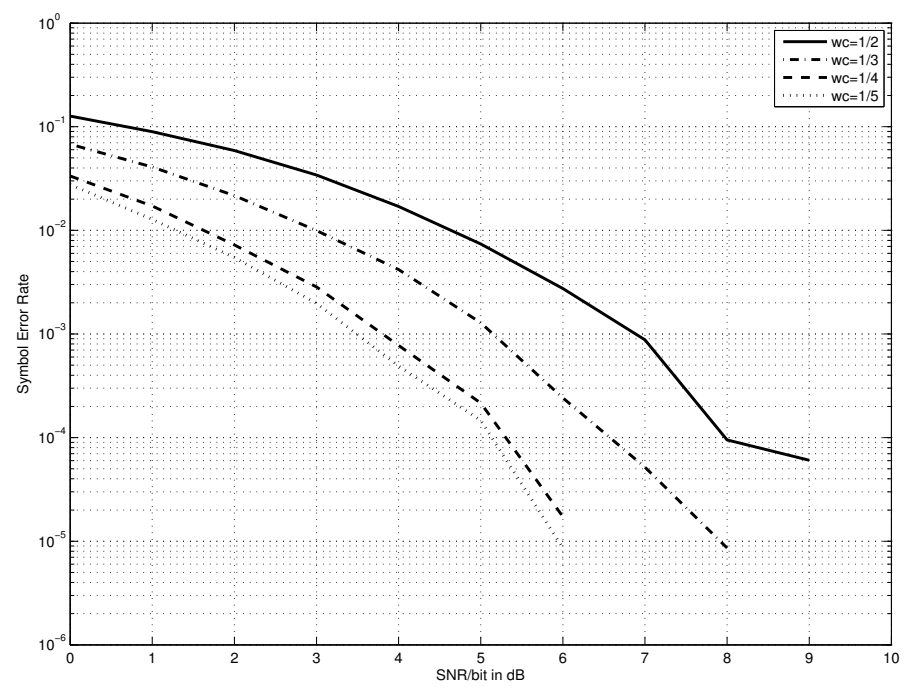

Figure 14. The BER performances of OFDM system elliptic filter (cut-off frequency varied)

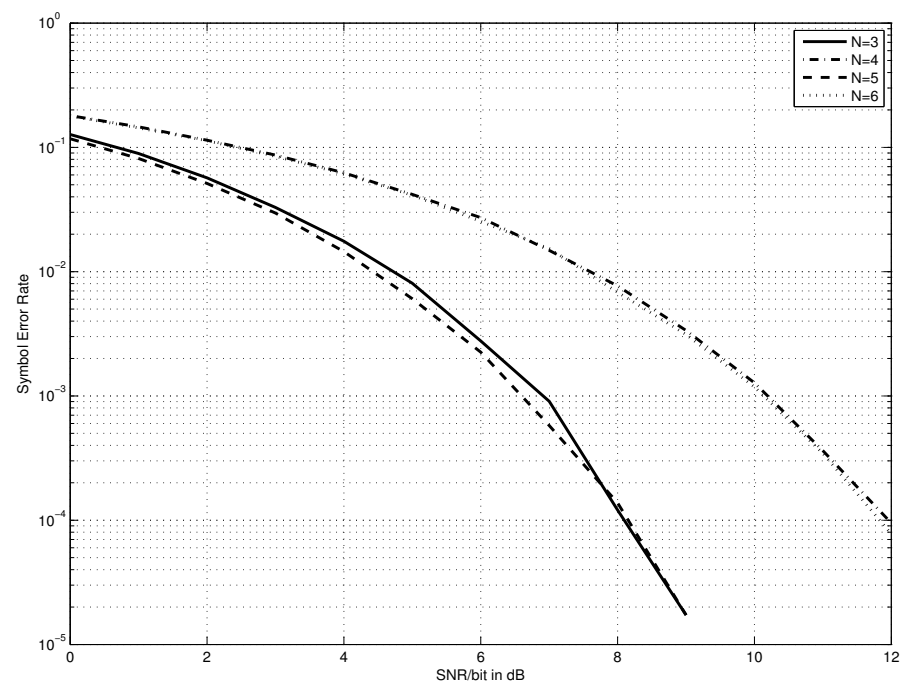

Figure 15. The BER performances of OFDM system using Chebyshev ( order varied). 
International Journal of Wireless \& Mobile Networks (IJWMN) Vol. 5, No. 3, June 2013

In the last investigation the Chebyshev filter is replaced by an elliptic filter by keeping other parameters same. We selected an elliptic filter with order 3. The pass band ripple was maintained at $0.5 \mathrm{~dB}$ and the stop band ripple was set to $10 \mathrm{~dB}$. The cut-off frequency was varied to different values. The simulation results are shown in Figure 14. It is depicted in the figure that the elliptic filter performs almost in the same way as the Butterworth filters does. For example, at the SNR of $4 \mathrm{~dB}$, the symbol error rates are $0.02,0.002,0.0004$ and 0.0003 for cut-off frequencies of $1 / 2,1 / 3,1 / 4$, and $1 / 5$ respectively. The figure also depicts that the elliptic filters with lower cut-off frequencies perform better than those of higher cut-off frequencies. Then the filter order was varied and the cut-off frequency was fixed to $1 / 2$. The simulation results are shown in Figure 15. It is shown that a higher order filter is desirable for OFDM receiver. But, the variation of the performances with the filter order is not significant in all cases.

\section{Conclusions}

In this paper the performance of OFDM based DVB-T receiver has been investigated. OFDM modulation scheme has drawn wide attentions by the researchers in academia and industry. Designing a good OFDM receiver design is still an open issue. It is shown in this paper that OFDM receiver performance depends heavily on the filter types. Different filters have been taken into account. It is shown that the filter types and some important filter parameters like filter order and cut-off frequency affect the OFDM receiver performance. It is widely accepted that the Butterworth filter is the most popular choice for OFDM receiver. But, Chebyshev filters and elliptic filters are also good candidate. It is also shown that OFDM receiver is more sensitive to Chebyshev and elliptic filters other than Butterworth filters.

\section{REFERENCES}

[1] Wu. Y, Hirakawa S., Reimers, U.H. and Whitaker, J., "Overview of Digital Television Development Worldwide”, In the Proceedings of IEEE, Vol. 94, January 2006, pp. 8-21

[2] Udelson, J.H., "The Great Television Race: A History of the American Television Industry", University of Alabama Press, 1982

[3] "The World in 2011: ICT Facts and Figures", Technical Report of International Telecommunication Union (ITU), October 2011.

[4] "Television History " available at http://www.tvhostory.tv

[5] www.dvb.org

[6] Peter, S., "DVB: Developing Global Television Standards for Today and Tomorrow", Technical Symposium at ITU , 2011

[7] Reimars, U.H., "DVB-The Family of International Standards for Digital Video Broadcasting”, In the Proceedings of IEEE, Vol. 94, No.1, pp. 173-182

[8] Ladebusch, U. and Liss, C.A., "Terrestrial DVB (DVB-T): A Broadcast Technology for Stationary Portable and Mobile Use", In the Proceedings of IEEE, Vol. 94, No.1, pp. 183-193

[9] Introduction to the DVB Project, DVB Fact Sheet, May 2013 available at http://www.dvb.org/technology/fact_sheets/DVB-Project_Factsheet.pdf

[10] ETSI EN 300 744, Digital Video Broadcasting (DVB): Measurement Guidelines for DVB system, v. 1.2.1 (2009-01) available at http://www.etsi.org/deliver/etsi_en/ 300700_300799/ 300744/ 01.06.01_60/ en_300744v010601p.pdf 
International Journal of Wireless \& Mobile Networks (IJWMN) Vol. 5, No. 3, June 2013

[11] Popovic, M. L.; Sunjevaric, M. M., and Vujasinovic, Z.J. , "Effects of DVB-H and 3G cellular networks integration", In the Proceedings of the $10^{\text {th }}$ International Conference on Telecommunications in Modern Satellite Cable and Broadcasting Services, Serbia, October 2011, pp. 641-644

[12] Nee, R.V. and Prasad, R. "OFDM Wireless Multimedia Communications”, Norwood, MA: Artech House, 2000

[13] Bingham, J.A.C. , "Multi-carrier modulation for data transmission: An idea whose time has come", IEEE Communications Magazine, Vol.28, No. 5, May 1990, pp. 5-14

[14] Pun,M.O., Morelli, M. and Kuo, J., "Multi-Carrier Techniques for Broadband Wireless Communications: A Signal Processing Perspective," Imperial College Press, 57 Shelton Street, Covent Garden, London WC2H 9HE

[15] Hirosaki, B. “An Analysis of automatic equalizer for orthogonally multiplexed QAM system”, IEEE Transaction on Communication, Vol. com 28, No. 1, 1980, pp. 73-83

[16] Vahlin, N., and Holte, N., “ Optimal finite duration pulses for OFDM", IEEE Transaction on Communications, Vol. 44, No. 11, January 1996, pp. 10-14

[17] Slepian, D. and Pollak,H., “ Prolate speroidal wave functions , Fourier analysis and uncertaintyI”, Bell System Technical Journal, Vol. 40, January 1961, pp. 43-63

[18] Chen,H.H., and CAI, X.D., " Optimization of Transmitter and Receiver Filters for OQAMOFDM Systems using Non-linear Programming”, IEEE Transaction on Communications, Vol. E80-B, No. 11, November 1997, pp. 1680-1687

[19] Aldis, J.P., Althoff, M.P. and Van-Nee, R., " Physical layer architecture and performance in the WAND user trial system", In the proceedings of ACTS Mobile Conference, Grenada, Spain, 1996, pp. 196-203

[20] Gudmundson, M. and Anderson, P.O., “Adjacent channel interference in an OFDM system”, In the Proceedings of IEEE Vehicular Technology Conference, 1996, pp. 918-922

[21] Cimini, L.J., "Analysis and simulation of a digital mobile channel using orthogonal frequency division multiplexing", IEEE Transaction on Communications, Vol. 33, 1985, pp. 665-675

[22] Khalid Aslam, Bodiuzzaman,M., Uddin J., and Kulesza, W, "Using Raised Cosine Filter to Reduce Inter- Symbol Interference in OFDM with BPSK technique", Computer Science Journal, Vol. 1, No. 2, August 2011, pp. 115-119

[23] Faulkner, M., “ The effects of Filtering on the performance of OFDM systems”, IEEE Transactions on Vehicular Technology, Vol. 49, No.5, September 2000, pp. 1877-1884

[24] Prafullah D. G. and Siddharth A. L., “PAPR Performance of OFDM system by using Clipping and Filtering”, International Journal of Advances in Engineering and Technology, Vol. 6, No. 2, May 2013, pp. 789-794

[25] Wang, L. and Tallanbara, C., "A simplified clipping and Filtering technique for PAR Reduction in OFDM systems”, IEEE Signal Processing Letters, Vol. 12, No. 6, June 2005, pp. 453-456

[26] Zhong, X., Qi. J, and Bao, J., “ Using clipping and filtering algorithm to reduce PAPR of OFDM system", In the Proceedings of the International Conference on Electronics, Communication, and Control, September 2011, Ningbo, China, pp. 1763-1766

[27] Aziz W., Ahmed E., Abbas G., Saleem, S., and Islam, Q. “PAPR Reduction in OFDM using Clipping and Filtering”, World Applied Sciences Journal, January 2012, Vol. 11, pp. 14951500

[28] Ho C.K., Sun S., and Farhang B., " Detrimental Effects of filtering in an OFDM system using pilot based channel estimation", In the Proceedings of the $13^{\text {th }}$ IEEE International Symposium on Personal, Indoor, and Mobile Radio Communication, September , 2002, Vol. 3, pp. 1316-1320

[29] Naveen V,.J., and Rajeswan K.R, “ ICI Reduction using Extended Kalman Filter in OFDM system”, International Journal of Computer Application, Vol. 17, No. 7, March 2011, pp. 15-22 
International Journal of Wireless \& Mobile Networks (IJWMN) Vol. 5, No. 3, June 2013

[30] Li X., and Cimini L.J, "Effects of clipping and filtering on the performance of OFDM", IEEE Communication Letter, Vol. 2, No.5, May 1998, pp. 131-138

[31] Klinler, F. and Scelze, H., “ Adjacent Channel Interference with Imperfect Anti-Aliasing Filtering", In the Proceedings of the $12^{\text {th }}$ International OFDM- Workshop, August 07, Hamburg, Germany

[32] Saritha , H.M. and Kulakarni, M. , “ A novel scheme using MLE with pulse shaping for ICI cancellation in OFDM system", In the Proceedings of International Conference on Communication and Informatics, January 2012, Coimbatore, India, pp. 1-5.

[33] Chang, S. and Powers E.J., "Cancellation of inter-carrier interference in OFDM systems using a nonlinear adaptive filter", In the Proceedings of IEEE International Conference on Communication, 2000, Vol. 2, pp. 1039-1043

[34] OFDM Simulation using Matlab available at http://www.ece.gatech.edu/research/_labs/_sarl/ tutorials / OFDM/Tutorial_web.pdf

[35] John G. Proakis and Dimitris G. Manolakis, "Digital Signal Processing : Principles, Algorithms, and Applications”, Fourth Edition, Prentice Hall, Upper Saddle River, New Jersey, USA

[36] Ingle, V.K. and Proakis J.G., "Digital Signal Processing using MATLAB", Thomson Bookware Companion Series

[37] Antoniou A., "Digital Signal Processing: Signals, Systems and Filters", McGraw Hill, New York, USA

[38] Mitra S.K., "Digital Signal Processing: A Computer Base Approach”, McGraw Hill, New York, USA

[39] Bose T., "Digital Signal and Image Processing", John Wiley and Sons, New Jersey, USA 